\title{
CRITICAL GROUPS HAVING CENTRAL MONOLITHS OF A NILPOTENT BY ABELIAN PRODUCT VARIETY OF GROUPS
}

\author{
BY
}

JAMES J. WOEPPEL

\begin{abstract}
Let $\mathfrak{R}$ be a variety of groups which has nilpotency class two and finite odd exponent. Let $\mathfrak{A}$ be an abelian variety of groups with finite exponent relatively prime to the exponent of $\mathfrak{R}$. The existence in the product variety $\mathfrak{R}$ of nonnilpotent critical groups having central monoliths is established. The structure of these critical groups is studied. This structure is shown to depend on an invariant, $k$. The join-irreducible subvariety of $\mathfrak{N} \mathfrak{A}$ generated by the nonnilpotent critical groups of $\mathfrak{R}$ having central monoliths is determined, in particular, for $k$ odd.
\end{abstract}

1. Introduction. (Throughout any terminology or notation not explained is described in Hanna Neumann's book [4].) Any nilpotent variety of nilpotency class two and finite exponent is of the form $\mathfrak{B}_{n} \wedge \mathfrak{A}_{n} \mathfrak{A}_{n^{\prime}} \wedge \mathfrak{N}_{2}$ (where $n^{\prime}$ divides $n)$; let $\mathfrak{R}_{\left(n, n^{\prime}\right)}$ denote this variety. The variety $\mathfrak{N}_{(q, q)} \mathfrak{A}_{m}$ is a subvariety of the product variety $\mathfrak{R}_{\left(n, n^{\prime}\right)} \mathfrak{A}_{m}$, if $q$ divides $n^{\prime}$. Thus we may assume that $n$ equals $n^{\prime}$ and that $n$ is a power of a prime. In [7], the product variety $\mathfrak{R}_{(q, q)} \mathfrak{A}_{m}$ was shown to be join-irreducible for $q$ a nontrivial power of an odd prime not dividing $m$. The critical groups generating these product varieties were also characterized in that paper. For distinct primes $p$ and $r$, satisfying $r$ dividing $p-1$, R. G. Burns [2] has obtained a complete description of the lattice of subvarieties of $\mathfrak{R}(p, p) \mathfrak{U}_{r}$.

Let $k$ be the smallest positive integer such that $m$ divides $p^{k}-1$ where $p$ is an odd prime not dividing the positive integer $m$, and let $q$ equal $p^{e}$. The joinirreducible subvariety of $\mathfrak{R}_{(q, q)} \mathfrak{A}_{m}$ generated by the nonnilpotent critical groups in this variety having central monoliths is determined in the case that $m$ does not divide $p^{k / 2}+1$.

The critical groups of the variety $\mathfrak{R}_{(q, q)} \mathfrak{A}_{m}$ having central monoliths are contained in the subvariety $\mathfrak{R}_{(q, q)} \mathfrak{A}_{m} \wedge \mathfrak{E}$, where $\mathfrak{E}$ denotes the variety of groups satisfying the law,

Received by the editors August 15, 1975.

AMS (MOS) subject classifications (1970). Primary 20E10; Secondary $20 \mathrm{~F} 25$.

Key words and phrases. Varieties of groups, join-irreducible varieties, Cross varieties, critical group, central monolith, nilpotent by abelian. 


$$
[[x, y],[u, t]]^{z}[[u, t],[x, y]]=1 .
$$

Thus the third subgroup in the derived series of a group in $\mathbb{E}$ is central. The following result is obtained in $\$ 4$ :

THEOREM I. The variety $\mathfrak{R}_{(q, q)} \mathfrak{A}_{m} \wedge \mathbb{\varepsilon}$ is join-irreducible, if $k$ is odd or if $k$ is even with $m$ not dividing $p^{k / 2}+1$, where $k$ is the smallest positive integer such that $m$ divides $p^{k}-1$.

The results of $\$ 4$ are not sufficient to resolve the case for $k$ even and $m$ dividing $p^{k / 2}+1$.

To establish Theorem I it is necessary to study in $\$ 2$ the structure of the nonnilpotent critical groups in $\mathfrak{N}_{(q, q)} \mathfrak{A}_{m}$ having central monoliths. A description of this structure is given in Theorems II and III.

THEOREM II. If $C$ is a nonnilpotent critical group in $\mathfrak{R}_{(q, q)} \mathfrak{A}_{m}$ having a central monolith, then

(i) the $p^{\prime}$-complement of the Fitting subgroup, Fit $C$, is cyclic, and

(ii) the exponent of the commutator subgroup, (Fit $C)^{\prime}$, of the Fitting subgroup equals the exponent of the Fitting subgroup.

The results of R. G. Burns [1] show that if $C$ is a critical group in $\mathfrak{R}_{(q, q)} \mathfrak{A}_{m}$ with the exponent of $C /$ Fit $C$ equaling $m$, then the Fitting subgroup is generated by $k$ or $2 k$ elements, where $k$ is the invariant defined above. The following theorem is also established in $\$ 2$ :

THEOREM III. Let $C$ be a nonnilpotent critical group in $\mathfrak{R}_{(q, q)} \mathfrak{A}_{m}$ having a central monolith. If Fit $C$ is generated by $k$ elements and $C / F i t ~ C$ has exponent $m$, then $k$ is even and $m$ divides $p^{k / 2}+1$.

It should be noted that if the word "critical" is replaced by "monolithic", the theorem remains true.

A nonnilpotent critical group in the variety $\mathfrak{R}_{(q, q)} \mathfrak{A}_{m}$ having a central monolith is constructed in \$3. The method of construction depends on the invariant $k$.

2. Structure of critical groups having central monoliths. Let $C$ be a nonnilpotent critical group in $\mathfrak{R}_{(q, q)} \mathfrak{A}_{m}$. Since $C$ is a monolithic group, the Fitting subgroup, Fit $C$, is a $p$-group where $q=p^{e}$. Thus $C$ is the semidirect product of Fit $C$ and the $p^{\prime}$-complement $D$ of Fit $C$ in $C$; that is, $C=($ Fit $C) \cdot D$. Set $F=$ Fit $C$ and $\Phi=\Phi$ (Fit $C$ ), the Frattini subgroup of Fit $C$.

By Maschke's theorem [3], the Frattini quotient group $F / \Phi$ viewed as a $G F(p) D$-module may be decomposed into the direct product of irreducible $G F(p) D$-modules, and by Burns' results there are at most two such irreducible $G F(p) D$-modules in this decomposition. Thus either $F / \Phi$ is a minimal normal subgroup of $C / \Phi$ or

$$
F / \Phi=N_{1} / \Phi \times N_{2} / \Phi
$$

where $N_{1} / \Phi$ and $N_{2} / \Phi$ are minimal normal subgroups of $C / \Phi$. Since $C$ is a 
critical group, $D$ is represented faithfully by conjugation on $F / \Phi$, and thus $D$ is embeddable in the direct product of two $m$-cycles, $C_{m} \times C_{m}$; see Burns [1].

Proof OF THEOREM II. For this proof set $m^{\prime}=\exp (D), t=\exp (F)$, and $t^{\prime}$ $=\exp \left(F^{\prime}\right)$. If $F / \Phi$ is a minimal normal subgroup in $C / \Phi$, then $D$ is cyclic because it is faithfully and irreducibly represented on $F / \Phi$. On the other hand, if $F / \Phi$ has (2.1) as its decomposition into irreducible $G F(p) D$-modules, then the centralizer of $N_{1} / \Phi, C_{D}\left(N_{1} / \Phi\right)$, in $D$ is trivial, because the monolith, $\left[N_{1}, N_{2}\right]^{t / p}$, of $C$ is centralized by $D$. Thus again $D$ is faithfully and irreducibly represented on $N_{1} / \Phi$, and so $D$ is cyclic; hence part (i) has been established.

Part (ii) is proved by contradiction. Assume that $t^{\prime}<t$. There are elements $z_{1}$ and $z_{2}$ in $F$ such that $F$ is the normal closure of the subgroup generated by $z_{1}$ and $z_{2}$ in $C$ (in some cases, one element would suffice). The subgroup $F$ is a regular $p$-group since $p$ is an odd prime. Thus either the order of $z_{1}$ or the order of $z_{2}$ is $t$. However, the powers $z_{1}^{t / p}$ and $z_{2}^{t / p}$ are central in $F$ as the following calculation shows: $\left[z_{i}^{t / p}, x\right]=\left[z_{i}, x\right]^{t / p}=1$ for any $x$ in $F$. Since $C$ is monolithic, $z_{1}^{t / p}$ and $z_{2}^{t / p}$ must be in the monolith. Hence $z_{1}^{t / p}$ and $z_{2}^{t / p}$ are centralized by $D$. Thus, $\left(z_{1} z_{1}^{-\alpha}\right)^{t / p}$ and $\left(z_{2} z_{2}^{-\alpha}\right)^{t / p}$ are trivial for $\alpha$ in $D$. However, if $\alpha$ is a generator of $D, F$ is the normal closure of the subgroup generated by $\left(z_{1} z_{1}^{-\alpha}\right)$ and $\left(z_{2} z_{2}^{-\alpha}\right)$ in $C$; that is, $F$ is generated by elements of order $t / p$ or less. This is a contradiction since $F$ is a regular $p$-group.

Theorem III shows that the structure of the nonnilpotent critical groups in $\mathfrak{R}_{(q, q)} \mathfrak{A}_{m}$ having central monoliths depends on the invariant $k$. If the exponent of the $p^{\prime}$-complement, $D$, of $F$ in $C$ is $m$, then the dimension of the irreducible $G F(p) D$-modules in the decomposition of $F / \Phi$ is $k$; this follows from M. F. Newman's results in [5].

ProOF OF THEOREM III. Let the critical group $C=F \cdot D$ as above with $t$ the exponent of $F$. The $p^{\prime}$-group $D$ has exponent $m$ by hypothesis and is cyclic by Theorem II, part (i); let $\alpha$ generate $D$. Also, $F / \Phi$ is an irreducible $G F(p) D$ module by hypothesis.

Suppose there is a $y$ in $F$ but not in $\Phi(F)$ such that some nontrivial pth power, $g$, of $y$ is in the central monolith. This implies that $\left(y y^{-\alpha}\right)^{g}$ is trivial, but the normal closure of the subgroup generated by $y y^{-\alpha}$ in $C$ is $F$; contradiction. Therefore $F / F^{p}$ is a nonabelian monolithic $p$-group; let $F^{*}$ denote the quotient group $F / F^{p}$. (Note that, $F$ is regular since $p>2$.)

Clearly $\Phi\left(F^{*}\right)=\left(F^{*}\right)^{\prime}$. The center, $Z\left(F^{*}\right)$, of $F^{*}$ also equals $\left(F^{*}\right)^{\prime}$ since $Z\left(F^{*}\right)$ is a proper characteristic subgroup of $F^{*}$. The order of $\left(F^{*}\right)^{\prime}$ is $p$ since it is the central monolith of $C /(F)^{p}$. Thus $F^{*}$ is an extra-special $p$-group [3, p. 183].

By [3, Theorem 6.5, p. 213], the order, $m$, of $\alpha$ divides $\left(p^{2 r}-1\right)$ with $0<2 r \leqslant k$, which contradicts the choice of $k$ unless $2 r=k$. Therefore $k$ is even and $m$ divides $\left(p^{k / 2}+1\right)$.

In the above proof it is sufficient to assume that $C$ is monolithic; the remaining hypothesis implies that $D$ is cyclic. The author has been able to establish the converse of Theorem III; however, the proof of this is too lengthy to include here. 
3. The existence of critical groups having central monoliths. Let $F_{k}$ be a relatively free group of rank $k$ of the variety $\Re_{(q, q)}$. In [7], the existence of an automorphism $\beta$ and a generating set $\left\{y_{1}, \ldots, y_{k}\right\}$ of $F_{k}$ such that $y_{1} \beta=y_{2}$, $\ldots, y_{k-1} \beta=y_{k}$ was established. Now let $F_{2 k}$ be a relatively free group of $\Re_{(q, q)}$ freely generated by $\left\{y_{11}, \ldots, y_{1 k}, y_{21}, \ldots, y_{2 k}\right\}$. There are automorphisms $\beta_{1}$ and $\beta_{2}$ of $F_{2 k}$ each of order $m$ such that $\beta_{i}$ fixes each $y_{j n}$ with $j \neq i$ and acts on $\left\{y_{i 1}, \ldots, y_{i k}\right\}$ as $\beta$ acts on $\left\{y_{1}, \ldots, y_{k}\right\}$ [7]. Let $B$ be the abelian subgroup of the automorphism group of $F_{2 k}$ generated by $\left\{\beta_{1}, \beta_{2}\right\}$, and let $V$ be the splitting extension of $F_{2 k}$ by $B$. The group $V$ generates the variety $\mathfrak{R}_{(q, q)} \mathfrak{A}_{m}[7]$.

$$
T=g p\left\langle\left[y_{i j}, y_{i n}\right],\left(\left[y_{1 j}, y_{2 j}\right]\left[y_{2 j}^{\beta_{2}^{-1}}, y_{1 j}^{\beta_{1}}\right]\right)^{\beta} \mid i=1,2 ; 1 \leqslant j, n \leqslant k ; \beta \in B\right\rangle
$$

defines a normal subgroup of $V$ contained in $\left(F_{2 k}\right)^{\prime}$. (It turns out that $V / T$ is a critical group generating the variety $\mathfrak{N}_{(q, q)} \mathfrak{A}_{m}[7]$.) Let $A$ denote the subgroup of $V / T$ generated by $\alpha=\beta_{1} \beta_{2}^{-1} T ; 2^{q}$ denote $F_{2 k} / T$; and $R$ denote the semidirect product of 2 by $A$. The commutator subgroup $\mathcal{Q}^{\prime}$ of $\mathcal{L}$ is homocyclic and is centralized by $A$. If $m=2$, then $\mathcal{Q}^{\prime}$ is cyclic and $R$ is monolithic.

Now, assuming $m \neq 2$, let $N$ be the complement of

$$
g p\left\langle\left[y_{11}, y_{21}^{\beta_{2}^{-1}}\right]\left[y_{21}, y_{11}^{\beta_{11}}\right] T\right\rangle
$$

in $\mathcal{Q}^{\prime}$ which is normal in $R$ given by D. R. Taunt's results [6]. The quotient group $C^{*}=R / N$ is monolithic with monolith $\left(2^{\prime} / N\right)^{q / p}$, since $2 / N$ is a regular p-group. For $m=2$, set $C^{*}=R$. If $k$ is odd, or if $k$ is even with $m$ not dividing $p^{k / 2}+1, C^{*}$ is a nonnilpotent critical group of $\mathfrak{R}_{(q, q)} \mathfrak{A}_{m}$ having a central monolith. If $k$ is even with $m$ dividing $p^{k / 2}+1$, then the subgroup of $C^{*}$ generated by $\alpha$ and $y_{11} y_{21} N$ is the desired critical group having a central monolith; denote this group by $C^{* *}$. That is, $C^{* *}=g p\left\langle\alpha, y_{11} y_{21} N\right\rangle$.

We now define inductively the word $\omega$ which is used in showing that the groups $C^{*}$ and $C^{* *}$ are critical groups. First let

$$
\mu_{2}\left(x_{1}, x_{2}\right)=\left[x_{1}, x_{2},\left(x_{2}^{-1} x_{1}\right)\right],
$$

and for $n$ in $\{3, \ldots, m\}$, put

$$
\mu_{n}\left(x_{1}, \ldots, x_{n}\right)=\left[\mu_{n-1}, x_{n},\left(x_{n}^{-1} x_{1}\right), \ldots,\left(x_{n}^{-1} x_{n-1}\right)\right] .
$$

Finally, set

$$
\omega=\left(\left[\mu_{m}\left(x_{1}, \ldots, x_{m}\right), \mu_{m}\left(z_{1}, \ldots, z_{m}\right)\right]\right)^{q / p} .
$$

The following substitution shows that $\omega$ is not a law of $C^{*}$ or $C^{* *}: x_{j}$ $\mapsto \alpha^{(j-1)}, z_{j} \mapsto \alpha^{(j-1)}, j=2, \ldots, m$, and $x_{1} \mapsto y_{11} y_{21} N, z_{1} \mapsto\left(y_{11} y_{21} N\right)^{\alpha}$.

THEOREM 3.1. The groups $C^{*}$ and $C^{* *}$ are critical. 
Proof. Since the groups $C^{*}$ and $C^{* *}$ are monolithic and since $\omega$ is not a law for $C^{*}$ or $C^{* *}$, to establish that $C^{*}$ and $C^{* *}$ are critical groups, we need only show by a result of Kovács and Newman (see 53.41 in [4]) that $\omega$ is a law in their proper subgroups. Only the proof for $C^{*}$ is given; the proof for $C^{* *}$ is similar. Let $2^{*}$ denote the Fitting subgroup of $C^{*}$; that is, $2^{*}=2 / N$.

Suppose $H$ is a subgroup of $C^{*}$ which does not have $\omega$ as a law. Since $\omega$ is not a law in $H$, there is a substitution for the $x_{1}, \ldots, x_{m}, z_{1}, \ldots, z_{m}$ in $\omega$ with values in $H$ such that $\omega$ is not the identity. Under this substitution $\mu_{m}\left(x_{1}, \ldots, x_{m}\right)$ and $\mu_{m}\left(z_{1}, \ldots, z_{m}\right)$ have values in $2^{*}$ outside of the Frattini subgroup, $\Phi\left(2^{*}\right)$. It is also clear that each of the values substituted for $x_{1}, \ldots, x_{m}$ (or for $z_{1}, \ldots, z_{m}$ ) lie in distinct cosets of $2^{*}$ in $C^{*}$. Hence the order of $H$ is divisible by $m$. Set $2_{H}=$ Fit $H$. The p-group $P$ $=2_{H}\left(2^{*}\right)^{p} /\left(2^{*}\right)^{p}$ is an extra-special $p$-group. Since $k$ is odd or $k$ is even with $m$ not dividing $p^{k / 2}+1, P / P^{\prime}$ cannot be an irreducible $G F(p) A$-module [3, p. 213]. Thus $2_{H} \Phi\left(2^{*}\right) / \Phi\left(2^{*}\right)$ is not an irreducible $G F(p) A$-module; hence $2_{H}=2^{*}$. Therefore $H$ is not a proper subgroup of $C^{*}$.

4. Proof of Theorem I. For notational convenience set $\mathfrak{U}=\mathfrak{R}_{(q, q)} \mathfrak{A}_{m} \wedge \mathfrak{c}$ and from $\$ 3, R=g p\left\langle\beta_{1} \beta_{2}^{-1} T, F_{2 k}\right\rangle / T$. The proof of Theorem 1 is accomplished by establishing the following three propositions:

(i) The group $R$ generates the variety $\mathfrak{u}$.

(ii) There is a proper subvariety of $\mathfrak{u}$ which contains any critical group $C$ of $\mathfrak{u}$ in which either (a) the exponent of the commutator subgroup of Fit $C$ is less than $q$, or (b) the order of $C /$ Fit $C$ is less than $m$.

(iii) For $k$ odd or $k$ even with $m$ not dividing $p^{k / 2}+1$, a nonnilpotent critical group $C$ having a central monolith generates the variety $\mathfrak{U}$ if the exponent of Fit $C$ is $q$ and $C /$ Fit $C$ has order $m$.

Proposition (i) is established by showing that any critical group $C$ of $\mathfrak{u}$ is a factor of a direct power of $R$. If $C$ is nilpotent this result is obvious. For any nontrivial divisor $m^{*}$ of $m$ and corresponding smallest positive integer $k^{*}$ such that $m^{*}$ divides $p^{k^{*}}-1$, there is, by Clifford's theorem [3], a subgroup of $R$ which has a corresponding structure like $R$ for $m^{*}$ and $k^{*}$. Hence we may assume that $C / F$ has order $m$, where as before $F=$ Fit $C$. It was shown in [7] that any critical group $C$ in $\mathfrak{N}_{(q, q)} \mathfrak{A}_{m}$ is a factor of $V$, the splitting extension of $F_{2 k}$ by $B$. There are three cases: $\operatorname{dim}(F / \Phi)=k, \operatorname{dim}(F / \Phi)=2 k$ with $m$ not dividing $p^{k / 2}+1$, and $\operatorname{dim}(F / \Phi)=2 k$ with $m$ dividing $p^{k / 2}+1$, where $\Phi=\Phi(F)$.

Let $F / \Phi$ have dimension $k$. In this case, it can be shown that $C$ is a factor of $V^{*}$, the splitting extension of $F_{k}$ by the $m$-cycle $\beta$ of $\$ 3$; in the same way that $C$ was shown to be a factor of $V$ in [7]. The group $V^{*}$ is isomorphic to the subgroup $\mathcal{V}^{*}$ of $V$ generated by $y_{11} y_{21}$ and $\beta_{1} \beta_{2}^{-1}$. Hence $C$ is a homomorphic image of $\mathcal{V}^{*}$, and clearly the intersection of $\mathcal{V}^{*}$ and $T$ is contained in the kernel of this homomorphism of $\mathscr{V}^{*}$ onto $C$. Thus $C$ is a factor of $R$.

Now suppose $\operatorname{dim}(F / \Phi)$ is $2 k$ and $m$ does not divide $p^{k / 2}+1$. Recall the minimal normal subgroups $N_{1} / \Phi$ and $N_{2} / \Phi$ of $C / \Phi$ in the decomposition (2.1) 
of $F / \Phi$ and the $p^{\prime}$-subgroup $D$ of $C$ in $\$ 2$. The quotient group

$$
\left(g p\left\langle N_{i}, D\right\rangle\right) F^{p} / F^{p}
$$

is monolithic, and so by Theorem III (see the note at the end of the proof of Theorem III), it must be metabelian. Thus $N_{1}$ and $N_{2}$ can be chosen so that $\left(N_{1}\right)^{\prime}$ and $\left(N_{2}\right)^{\prime}$ are trivial. The critical group $C$ is a homomorphic image of the subgroup $\widetilde{V}$ of $V$ generated by $F_{2 k}$ and $\beta_{1} \beta_{2}^{-1}$. The kernel of this homomorphism contains $T$, since $\left(N_{1}\right)^{\prime}$ and $\left(N_{2}\right)^{\prime}$ are trivial and since the monolith of $C$ is central. Therefore $C$ is a factor of $R$.

In the third case where $\operatorname{dim}(F / \Phi)$ is $2 k$ but $k$ is even with $m$ dividing $p^{k / 2}+1, N_{1}$ and $N_{2}$ need not be abelian. The critical group $C$ is again a homomorphic image of $\checkmark$. The group $\widetilde{V}$ can be embedded in the direct product $V^{*} \times \checkmark / T_{1} \times V^{*}$, where $T_{1}$ is the normal closure of the subgroup of $V$ generated by commutators of the form $\left[y_{1 j}, y_{1 j^{\prime}}\right]$ and $\left[y_{2 j}, y_{2 j^{\prime}}\right]$ for $1 \leqslant j, j^{\prime}$ $\leqslant k$ in $V$. It is easy to verify that $V^{*}$ is isomorphic to the subgroup of $\checkmark / T_{1}$ generated by $\beta_{1} \beta_{2}^{-1}$ and $y_{11} y_{21} T_{1}$; hence $C$ is a factor of the direct power $\checkmark / T_{1} \times \mathfrak{V} / T_{1} \times \checkmark / T_{1}$. Since the monolith of $C$ is central, $C$ is a factor of the direct power $R \times R \times R$.

The proper subvariety of proposition (ii) is the subvariety of $\mathfrak{u}$ determined by $\omega$. To establish (ii) all that needs to be shown is that if $C$ is a critical group of $\mathfrak{U}$ which does not have $\omega$ as a law, then the exponent of (Fit $C)^{\prime}$ is $q$ and $C /$ Fit $C$ has order $m$. That the exponent of the commutator subgroup is $q$ is obvious. The argument that $C /$ Fit $C$ has order $m$ is essentially the argument in the proof of Theorem 3.1, that the order of $H$ is divisible by $m$. Therefore proposition (ii) is established.

Let $C$ be a critical group of $\mathfrak{u}$ satisfying the hypothesis of proposition (iii). The critical group $C$ is a homomorphic image of $R$ by the proof of (i). If $\mathscr{K}$ is the kernel of this homomorphism, then $\mathcal{K}$ is contained in the commutator subgroup of $F_{2 k} / T$ since $C /$ Fit $C$ has order $m$. The kernel $\mathcal{K}$ cannot contain the socle, $S$, of $R$ because the exponent of (Fit $C)^{\prime}$ is $q$ by Theorem II(ii). Thus, $R$ must lie in the variety generated by $C$, if the socle $S$ of $R$ is minimal characteristic. By proposition (i), this implies that $C$ generates the variety $\mathfrak{U}$. Therefore the final step in the proof is to show that the socle, $S$, of $R$ is minimal characteristic in $R$.

To establish that $S$ is minimal characteristic, it is necessary to study the action of conjugation by $\beta_{2}$ on the group $R$. First, the subgroup $\checkmark$ of $V$ generated by $\beta_{1} \beta_{2}^{-1}$ and $F_{2 k}$ is clearly normalized by $\beta_{2}$, and the subgroup $T$ of $F_{2 k}$ is also normalized by $\beta_{2}$. Thus conjugation by $\beta_{2}$ induces an automorphism on $R$. Now consider the action of conjugation by $\beta_{2}$ on the element $\left[y_{11}, y_{21}\right]^{q / p} T$ in the socle of $R$. Clearly the elements $\left(\left[y_{11}, y_{21}\right]^{q / p} T\right)^{\beta}$ for $\beta \in g p\left\langle\beta_{1} \beta_{2}^{-1}, \beta_{2}\right\rangle$ generate the socle, $S$, of $R$. Therefore $S$ is minimal characteristic in $R$. 


\section{REFERENCES}

1. R. G. Burns, Verbal wreath products and certain product varieties of groups, J. Austral. Math. Soc. 7 (1967), 356-374. MR 36 \#249.

2. Univ., Canberra, Australia, 1966.

3. Daniel Gorenstein, Finite groups, Harper and Row, New York, 1968. MR 38 \#229.

4. Hanna Neumann, Varieties of groups, Ergebnisse der Mathematik und ihrer Grenzgebiete, Band 37, Springer-Verlag, New York, 1967. MR 35 \#6734.

5. M. F. Newman, On a class of metabelian groups, Proc. London Math. Soc. (3) 10 (1960), 354-364. MR 22 \#8074.

6. D. R. Taunt, On A-groups, Proc. Cambridge Philos. Soc. 45 (1949), 24-42. MR 10, 351.

7. James J. Woeppel, Join-irreducible Cross product varieties of groups, Trans. Amer. Math. Soc. 200 (1974), 141-148.

Division of Natural Sciences, Indiana University Southeast, New Albany, Indiana 47150 